\title{
Study protocol: baby-OSCAR trial: Outcome after Selective early treatment for Closure of patent ductus ARteriosus in preterm babies, a multicentre, masked, randomised placebo-controlled parallel group trial
}

\author{
Samir Gupta ${ }^{1 *}$, Edmund Juszczak ${ }^{2,3}$, Pollyanna Hardy ${ }^{2,4}$, Nimish Subhedar ${ }^{5}$, Jonathan Wyllie ${ }^{6}$, Wilf Kelsall?,
} Sunil Sinha ${ }^{6}$, Sam Johnson ${ }^{8}$, Tracy Roberts ${ }^{4}$, Elisabeth Hutchison², Justine Pepperell ${ }^{2}$, Louise Linsell ${ }^{2}$, Jennifer L. Bell ${ }^{2}$, Kayleigh Stanbury' ${ }^{2}$, Marketa Laube ${ }^{2}$, Clare Edwards' ${ }^{2}$, David Field ${ }^{8}$ and on behalf of the 'The Baby-OSCAR Collaborative Group'

\begin{abstract}
Background: The question of whether to treat patent ductus arteriosus (PDA) early or wait until symptoms appear remains high on the research agenda for neonatal medicine. Around 7000 extremely preterm babies under 29 weeks' gestation are born in the UK every year. In 40\% of cases the PDA will fail to close spontaneously, even by 4 months of age. Untreated PDA can be associated with several serious and life-threatening short and long-term complications. Reliable data to support clinical decisions about PDA treatment are needed to prevent serious complications in high risk babies, while minimising undue exposure of infants. With the availability of routine bedside echocardiography, babies with a large PDA can be diagnosed before they become symptomatic.

Methods: This is a multicentre, masked, randomised, placebo-controlled parallel group trial to determine if earlytargeted treatment of a large PDA with parenteral ibuprofen in extremely preterm babies $\left(23^{+0}-28^{+6}\right.$ weeks' gestation) improves short and long-term health and economic outcomes. With parental informed consent, extremely preterm babies (born between $23^{+0}-28^{+6}$ weeks' gestation) admitted to tertiary neonatal units are screened using echocardiography. Babies with a large PDA on echocardiography, defined by diameter of at least $1.5 \mathrm{~mm}$ and unrestricted pulsatile PDA flow pattern, are randomly allocated to either ibuprofen or placebo within $72 \mathrm{~h}$ of birth. The primary endpoint is the composite outcome of death by 36 weeks' postmenstrual age or moderate or severe bronchopulmonary dysplasia (BPD) at 36 weeks postmenstrual age.

\footnotetext{
* Correspondence: samir.gupta@durham.ac.uk

'University Hospital of North Tees, Hardwick Road, Stockton-On-Tees TS19

$8 \mathrm{PE}, \mathrm{UK}$

Full list of author information is available at the end of the article
}

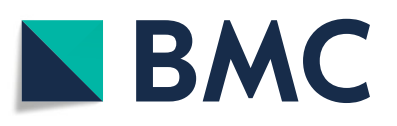

(- The Author(s). 2021, corrected publication 2021. Open Access This article is licensed under a Creative Commons Attribution 4.0 International License, which permits use, sharing, adaptation, distribution and reproduction in any medium or format, as long as you give appropriate credit to the original author(s) and the source, provide a link to the Creative Commons licence, and indicate if changes were made. The images or other third party material in this article are included in the article's Creative Commons licence, unless indicated otherwise in a credit line to the material. If material is not included in the article's Creative Commons licence and your intended use is not permitted by statutory regulation or exceeds the permitted use, you will need to obtain permission directly from the copyright holder. To view a copy of this licence, visit http://creativecommons.org/ licenses/by/4.0/. The Creative Commons Public Domain Dedication waiver (http://creativecommons.org/publicdomain/zero/1. 0/) applies to the data made available in this article, unless otherwise stated in a credit line to the data. 
(Continued from previous page)

Discussion: Prophylactic pharmacological treatment of all preterm babies unnecessarily exposes them to potentially serious side effects of drug treatment, when their PDA may have closed spontaneously. However, delaying treatment until babies become symptomatic could result in loss of treatment benefit as irreversible damage may have already been done.

Targeted, early pharmacological treatment of PDA in asymptomatic babies has the potential to overcome the disadvantages of both prophylactic (overtreatment) and symptomatic approaches (potentially too late). This could result in improvements in the clinically important short-term clinical (mortality and moderate or severe BPD at 36 weeks' postmenstrual age) and long-term health outcomes (moderate or severe neurodevelopment disability and respiratory morbidity) measured at 2 years corrected age.

Trial registration: ISRCTN84264977. Date assigned: 15/09/2010.

Keywords: Newborn, Patent ductus arteriosus, PDA, Echocardiography, Preterm, Ibuprofen, Bronchopulmonary dysplasia

\section{Background}

The Ductus Arteriosus (DA) is a vessel that allows blood from the right ventricle to bypass the fetal lungs to the placenta. In term babies it closes spontaneously after birth when breathing is established and is structurally closed after a few days. However, in a large number of preterm babies, the vessel does not close spontaneously resulting in a condition known as Patent Ductus Arteriosus (PDA). Around 7000 extremely preterm babies (< 29 weeks' gestation) are born in the UK every year. In $40 \%$ the PDA will fail to close spontaneously, even by 4 months of age [1].

PDA is associated with a number of serious and lifethreatening short and long-term complications. Complications of prematurity such as low blood pressure (hypotension), bleeding in the lungs (pulmonary haemorrhage) and brain (intraventricular haemorrhage (IVH)) present soon after birth. Other systemic complications such as necrotising enterocolitis (NEC) and bronchopulmonary dysplasia (BPD) present before discharge, and long-term health problems such as neurodevelopmental disability and chronic respiratory problems can be associated with PDA. The persistence of PDA has also been reported to be associated with an 8-fold increase in neonatal mortality $[2,3]$. Additionally, it places a significant financial burden on the National Health Service (NHS).

Historically, clinicians who have been concerned about the symptomatic PDA or complications of prematurity have attempted to close PDA utilising medical (pharmacological) or surgical treatment. Traditionally, medical treatment is instituted as prophylactic treatment (within $24 \mathrm{~h}$ of birth); early symptomatic treatment (usually 3-7 days after birth) and late symptomatic treatment (after one week of age). Prophylactic pharmacological treatment of all preterm babies unnecessarily exposes a large proportion of them to the potentially serious side effects of drug treatment, when their PDA would have closed spontaneously. Symptomatic treatment on the contrary delays treatment while waiting for symptoms to appear and could result in a loss of treatment benefit as damage may have already been done.

Moreover, the practice of a conservative approach of not treating, seems to originate from uncertainty regarding the management of PDA rather than evidence favouring no intervention. This is because most studies conducted to date have involved more mature preterm babies (over $1000 \mathrm{~g}$ or 28 weeks' gestation) whose PDA is more likely to close spontaneously. In addition, the studies were largely designed to assess PDA closure rates rather than assessing clinically important outcomes.

It is now suggested that large PDA on echocardiography in first $72 \mathrm{~h}$ (those with a diameter of $\geq 1.5 \mathrm{~mm}$ ) through which blood flow is pulsatile and unrestricted are less likely to close spontaneously. Targeted early treatment of a large PDA whilst asymptomatic has the potential to overcome the disadvantages of both the prophylactic and symptomatic approaches. Although clinical detection of PDA whilst asymptomatic is challenging, it can be assessed using bedside echocardiography.

Non-steroidal anti-inflammatory drugs, especially indomethacin and ibuprofen have been widely used for the treatment of PDA. Short term efficacy of indomethacin and ibuprofen are equivalent in the treatment of PDA [4]. Ibuprofen however appears to reduce the risk of NEC and is associated with fewer clinical gastrointestinal and renal side effects compared to indomethacin; hence it is the drug of choice for this trial. Paracetamol has also been recently reported in case studies for closure of symptomatic PDA but further research needs to be done to establish its safety \& effectiveness for early treatment of PDA [5].

The current literature falls short of providing substantive evidence on the management of PDA among extreme preterm babies leading to uncertainty and heterogeneity in clinical practices. 
A recent cohort study reported the presence of a large PDA (defined as a PDA dimension of $\geq 1.5 \mathrm{~mm}$ ) on day 3 in babies born before 28 weeks' gestation was associated with a threefold increase in odds of death or severe morbidity compared with neonates without PDA (Odds Ratio (OR) 3.4; 95\% Confidence Interval (CI) 1.1 to 11.0). Neonates with a large PDA were also reported to have increased odds of IVH (OR 4.2; 95\% CI 1.3 to 14.0) and BPD (OR 3.7; 95\% CI 1.0 to 14.0) compared with neonates with no PDA [6]. In preclinical trials, pharmacologic PDA closure is reported to improve alveolarisation and minimise the impaired postnatal alveolar development that is the pathologic hallmark of "new bronchopulmonary dysplasia (BPD)" [7]. An early selective treatment approach for closure of a PDA is suggested to trial its effect on BPD and mortality, which is the hypothesis of this trial.

We aim to determine if the selective treatment of echocardiographically confirmed large PDA in extremely preterm babies with ibuprofen within $72 \mathrm{~h}$ of birth reduces the incidence of death by 36 weeks postmenstrual age, or moderate or severe BPD at 36 weeks postmenstrual age.

\section{Methods/design}

\section{Aim \& Design}

This is a multicentre, masked, randomised, placebocontrolled parallel group trial to determine if the selective treatment of a large PDA with ibuprofen within $72 \mathrm{~h}$ of birth in extremely preterm babies $\left(23^{+0}-28^{+6}\right.$ weeks' gestation) reduces the incidence of death by 36 weeks postmenstrual age or moderate to severe bronchopulmonary dysplasia (BPD) at 36 weeks postmenstrual age. In addition, it assesses other secondary outcomes and health outcomes at 2 years corrected age including survival without moderate or severe neurodevelopmental disability and survival without respiratory morbidity.

An economic evaluation will be carried out from the perspective of the health service. It will take the form of a cost-effectiveness analysis presented in terms of cost per major outcome averted. The incremental cost estimate for statistically significant differences in the prespecified outcomes in primary and subgroup analyses would be computed.

Data are collected from hospital records and recorded on trial-specific case report forms (CRFs).

The main trial was preceded by an internal pilot phase which was used to assess the suitability of trial procedures and likelihood of recruitment targets being achieved. The Trial Steering Committee (TSC) reviewed pilot data and made recommendations regarding continuation. Data collected from the internal pilot phase of the trial will be included in the final analysis. The entire trial aims to recruit a total of 730 extremely preterm babies.

All enrolled babies are followed up at 2 years of age corrected for prematurity. Further longer-term follow-up at primary school age may be considered but will require separate funding. This may be undertaken as an amendment to this trial or as a separate application depending on the circumstances at the time.

The design of the trial is summarised in Fig. 1.

\section{Setting \& participants}

The trial is recruiting in 32 neonatal units. Only units that were in equipoise for the treatment of PDA, and agreed to perform echocardiograms within $72 \mathrm{~h}$ of birth to confirm the presence of a large PDA were selected.

\section{Inclusion criteria}

- Born at $23^{+0}-28^{+6}$ weeks' gestation

- Less than $72 \mathrm{~h}$ old

- Confirmed by echocardiography to have a large PDA which

- is at least $1.5 \mathrm{~mm}$ in diameter (determined by gain optimised colour Doppler), And

- has unrestrictive pulsatile (left to right) flow (ratio of flow velocity in PDA Maximum $\left(\mathrm{V}_{\max }\right)$ to Minimum $\left.\left(\mathrm{V}_{\min }\right)>2: 1\right)$ ) or, growing flow pattern $(<30 \%$ right to left), and no clinical concerns of pulmonary hypertension

In addition:

- The responsible clinician is uncertain about whether the baby might benefit from treatment to close the PDA

- Written informed consent is obtained from the parent(s).

\section{Exclusion criteria}

Babies will be excluded from participation in the trial if they have:

- No realistic prospect of survival

- Severe congenital anomaly

- Clinical or echocardiography suspicion of congenital structural heart disease that contraindicates treatment with ibuprofen

- Other conditions that would contraindicate the use of ibuprofen (active bleeding especially intracranial or gastrointestinal bleeding, coagulopathy, thrombocytopenia (platelet count $<50,000$ ), renal failure, life threatening infection, pulmonary 


\section{Inclusion criteria}

- Born at $23^{+0}$ to $28^{+6}$ weeks of gestation

- Less than 72 hours old

- Confirmed by echocardiography to have a large PDA which

- is at least $1.5 \mathrm{~mm}$ in diameter (determined by gain optimised colour Doppler), and

- has unrestrictive pulsatile (left to right) flow (ratio of flow velocity in PDA Maximum $\left(\mathrm{V}_{\max }\right)$ to Minimum $\left(\mathrm{V}_{\min }\right)>$ $2: 1)$ ) or, growing flow pattern ( $<30 \%$ right to left), and no clinical concerns of pulmonary hypertension

- The responsible clinician is uncertain about whether the baby might benefit from treatment to close the PDA

- Written informed consent has been obtained from the parent(s)

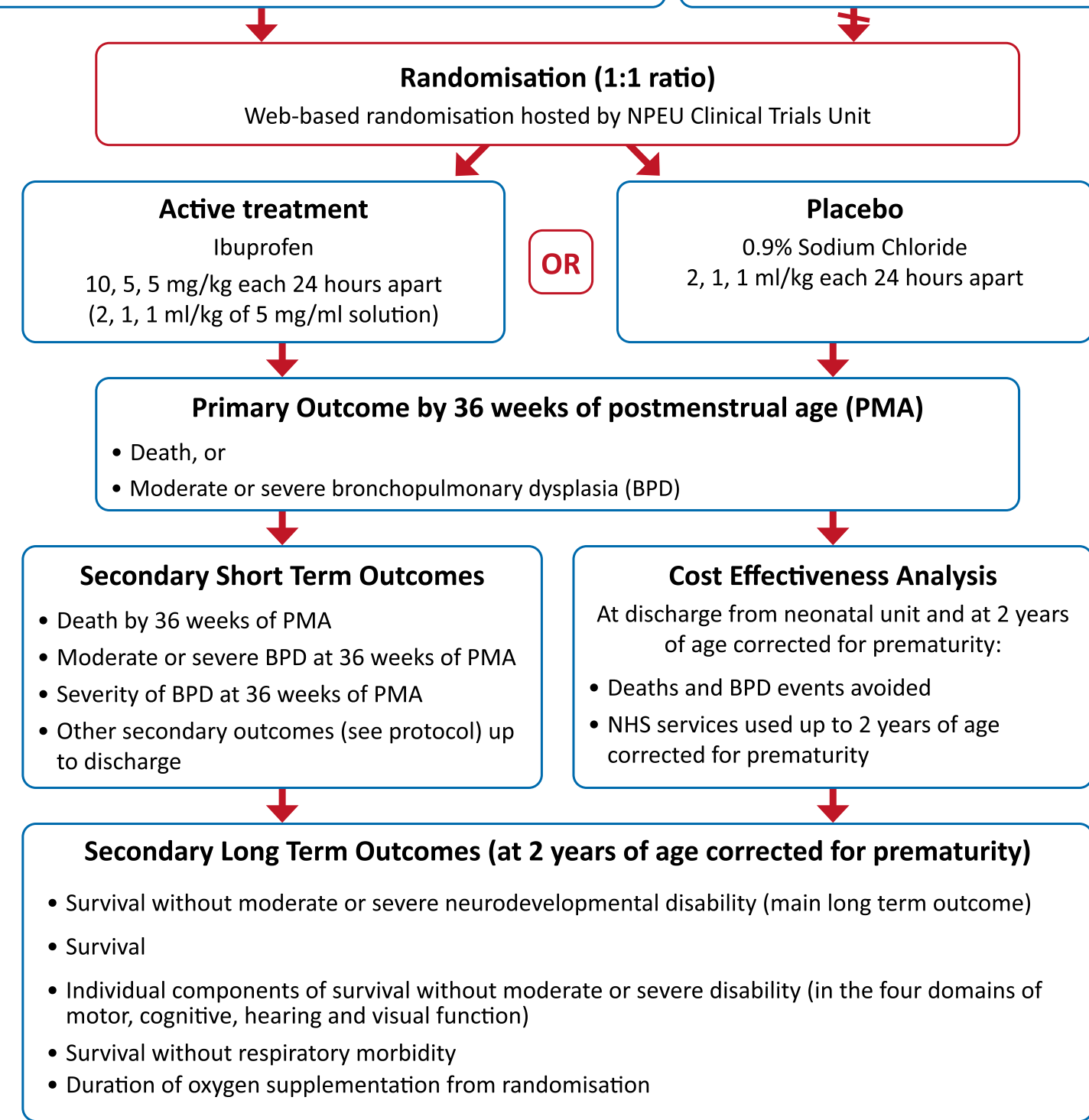

\section{Exclusion criteria}

- No realistic prospect of survival

- Severe congenital anomaly

- Clinical or echocardiography suspicion of congenital structural heart disease that contraindicates treatment with ibuprofen

- Contraindication to use of ibuprofen

- Indomethacin, ibuprofen, or paracetamol administration after birth

Fig. 1 Baby-OSCAR Participant Flow Diagram 
hypertension, known or suspected necrotising enterocolitis (NEC))

- Indomethacin, ibuprofen, or paracetamol administration after birth

Schedule of study procedures

\begin{tabular}{|c|c|c|c|c|c|c|}
\hline \multirow[t]{2}{*}{ Procedure } & \multicolumn{6}{|c|}{ Baby Hospitalisation } \\
\hline & Screening ${ }^{1}$ & $\begin{array}{l}\text { Trial Entry } \\
\text { and } \\
\text { Treatment } \\
\text { (days 1-3) }\end{array}$ & $\begin{array}{l}\text { Up to } 7 \\
\text { days after } \\
\text { trial } \\
\text { medication }\end{array}$ & $\begin{array}{l}3 \\
\text { weeks } \\
\text { of age }\end{array}$ & $\begin{array}{l}36 \\
\text { weeks } \\
\text { PMA }^{10}\end{array}$ & Discharge \\
\hline Demography ${ }^{9}$ & & $\mathrm{X}$ & & & & $\mathrm{x}$ \\
\hline $\begin{array}{l}\text { Echocardiogram/ } \\
\text { Colour Doppler }^{8}\end{array}$ & $\mathrm{x}$ & & & $\mathrm{x}$ & & \\
\hline $\begin{array}{l}\text { Confirmation of } \\
\text { Eligibility }\end{array}$ & $x$ & & & & & \\
\hline Consent & & $\mathrm{x}$ & & & & \\
\hline Randomisation $^{2}$ & & $\mathrm{x}$ & & & & \\
\hline $\begin{array}{l}\text { Ibuprofen/ } \\
\text { Placebo Dosing }\end{array}$ & & $\mathrm{x}$ & $\mathrm{x}$ & & & \\
\hline $\begin{array}{l}\text { IVH / PVL } \\
\text { ultrasound } \\
\text { scans }{ }^{10}\end{array}$ & & & $\mathrm{x}$ & & $x$ & \\
\hline NEC & & & & & & $x$ \\
\hline $\begin{array}{l}\text { Oxygen } \\
\text { Reduction Test }\end{array}$ & & & & & $\mathrm{x}$ & \\
\hline $\mathrm{SAEs}^{4}$ & & $\mathrm{X}$ & $\mathrm{x}$ & & & \\
\hline $\begin{array}{l}\text { Concomitant } \\
\text { Medication }^{5}\end{array}$ & $\mathrm{x}$ & $\mathrm{x}$ & & & & \\
\hline
\end{tabular}

Infant at 2 Years Corrected Age ${ }^{6,7}$

Demography $^{9} \quad \mathrm{X}$

Visual Assessment ${ }^{6}$

$\mathbf{X}$

Hearing Assessment ${ }^{6}$

Motor Assessment ${ }^{6}$

Respiratory Assessment ${ }^{7}$

Cognitive Assessment ${ }^{9}$

${ }^{1}$ Screening assessments to be completed sufficiently in advance to enable randomisation and dosing within 72 hours of birth. If consent cannot be obtained before echocardiographic evaluation for eligibility, echocardiographic assessment should continue, and consent obtained when possible if a baby is deemed eligible.

${ }^{2}$ Randomisation to be completed sufficiently in advance to enable starting IMP dosing within $72 \mathrm{~h}$ of birth.

${ }^{3}$ Initial trial drug administrations to be given soon after randomisation, after 6 hours of age and within 72 hours of birth. Subsequent doses to be administered 24 hours after the initial dose.

${ }^{4}$ Only adverse events which are serious will be recorded from first dose until 7 days after trial medication. Only unforeseeable SAEs will be reported. ${ }^{5}$ Concomitant medications to be recorded only in relation to unforeseeable SAEs. In the event of an unforeseeable SAE all concomitant medication, including medication given to the baby's mother, 7 days prior to the onset of the event to the time of its resolution must be recorded on the SAE form. ${ }^{6}$ Gross motor, cognitive, visual and hearing function will be assessed using the PARCA-R questionnaire, expanded to include questions to assess visual and hearing function.

${ }^{7}$ Respiratory assessments will be performed using a separate validated questionnaire. There will be no requirement for the infants to be assessed for respiratory and/or other neurodevelopmental functions by medically qualified personnel.

${ }^{8} \mathrm{An}$ echocardiogram scan will be performed when the baby reaches around 3 weeks of age (range of 18-24 days) or at hospital discharge if discharged earlier.
${ }^{9}$ Demography and medications at 2 years will be assessed through the PARCA$\mathrm{R}$ and other questionnaires.

${ }^{10}$ If a baby transfers from the recruiting site to a continuing care site for ongoing care details of any scan would be helpful.

\section{Primary outcome}

The primary outcome is defined as a composite outcome of incidence of death by 36 weeks postmenstrual age, or moderate or severe BPD at 36 weeks postmenstrual age shown in Table 1 .

The need for oxygen is subjective and hence oxygen dependency is confirmed using an 'oxygen reduction test'. This is based on the threshold at which the baby is able to maintain oxygen saturations $\geq 91 \%$ whilst breathing in air or at a given minimum $\mathrm{FiO}_{2}$. Babies unable to achieve this are considered to be oxygen dependent. This test only applies to those babies whose oxygen requirements are $<0.3$, or low flow oxygen $<1.1$ $\mathrm{L} / \mathrm{min}$, and who have not received any additional respiratory support in the previous $24 \mathrm{~h}$. Babies outside of this are not tested, but data on their oxygen requirements will be collected.

\section{Secondary outcomes \\ Short term outcomes}

- Death by 36 weeks postmenstrual age

- Moderate or severe BPD at 36 weeks postmenstrual age

- Severity of BPD at 36 weeks postmenstrual age (see table in Section 6.5)

Incidence or duration of the following up to discharge:

- Severe intraventricular haemorrhage (IVH) (grade III/IV with ventricular dilatation or intraparenchymal abnormality)

- Cystic periventricular leukomalacia (PVL)

- Non-cystic PVL

- Hydrocephalus

- Babies treated for retinopathy of prematurity (ROP)

- Significant pulmonary haemorrhage (fresh blood in endotracheal tube with increase in respiratory support)

- Treated for pulmonary hypertension with pulmonary vasodilator

- NEC definitive and/or complicated (Bell stage II and above) confirmed by radiology and/or histopathology

- NEC requiring surgery

- Gastrointestinal bleeding (leading to investigation or clinical treatment) within 7 days of the first dose of trial drug administration

- Spontaneous intestinal perforation 
Table 1 Severity-Based Diagnostic Criteria for BPD

\begin{tabular}{ll}
\hline Time point of & 36 weeks postmenstrual age \\
assessment: & \\
Therapy with oxygen $>$ & $21 \%$ and/or respiratory support for $\geq 28$ days and the following: \\
Mild BPD; & Baby is breathing room air \\
Moderate BPD; & Baby is in $22-29 \%$ oxygen, or $0.01-1.0 \mathrm{~L} / \mathrm{min}$ \\
Severe BPD; & $\begin{array}{l}\mathrm{FiO}_{2} \geq 0.3, \text { or low flow oxygen } \geq 1.1 \mathrm{~L} / \mathrm{min}, \text { or the baby is receiving any respiratory support (ventilation, CPAP, or high flow } \\
\text { oxygen therapy) to achieve saturations of } \geq 91 \%\end{array}$ \\
\hline
\end{tabular}

- Closed or non-significant PDA $(<1.5 \mathrm{~mm})$ at around 3 weeks of age (range of 18-24 days), confirmed by $\mathrm{ECHO}$

- $\mathrm{PDA} \geq 1.5 \mathrm{~mm}$ at around 3 weeks of age (range of 18-24 days)

- Medical open-label treatment of a symptomatic PDA with a COX inhibitor

- Open-label treatment of a symptomatic PDA by surgical treatment

- Administration and duration of inotropic support

- Total duration of respiratory support

a) Invasive ventilation through an endotracheal tube

b) Non-invasive support through nasal CPAP, nasal ventilation, humidified high flow nasal cannula therapy, or low flow oxygen $\geq 1.1 \mathrm{~L} / \mathrm{min}$

- Discharge home on oxygen

- Duration of initial hospitalisation (birth to discharge home)

- Postnatal steroid use for chronic lung disease

- Tolerance of ibuprofen treatment within the foreseeable SAE reporting range, described in the protocol, section 9.1.4

- Weight gain: a change in z score between birth and discharge (or death if sooner)

- Head circumference: a change in head size z score between randomisation and discharge (or death if sooner)

Long term outcomes assessed at 2 years of age corrected for prematurity

- Survival without moderate or severe neurodevelopmental disability (main long-term outcome)

- Survival

- Individual components of survival without moderate or severe neurodevelopmental disability (in the four domains of motor, cognitive, hearing and visual function). Cognitive disability will be assessed by determining the standardised non-verbal cognitive subscale and language subscale scores obtained through the Parent Report of Cognitive Abilities-
Revised (PARCA-R) [8] assessment. The PARCA-R assessment will be adapted to include questions to assess gross motor, hearing and visual function.

- Survival without respiratory morbidity. Respiratory morbidity will be assessed by the need for oxygen or respiratory support; presence of persistent cough and/or wheeze; need for regular treatment for respiratory illness; unscheduled attendances at hospital/GP; readmission to hospital for respiratory problems

- Duration of oxygen supplementation from randomisation

A cost-effectiveness analysis will be conducted of deaths and BPD events avoided and national health services used up to 2 years of age corrected for prematurity.

\section{Process outcomes}

- Number of doses of trial medication received

- Adherence to protocol (e.g. protocol violations, incidence of non-symptomatic open-label treatment etc.)

- Study withdrawals

\section{Screening and recruitment}

Extremely preterm babies potentially suitable for the trial are identified by the healthcare team within the neonatal unit. Information about the trial may be provided antenatally or soon after birth. Parent(s) are approached for consent after birth followed by echocardiographic evaluation for eligibility. However, if consent cannot be obtained echocardiographic assessment should continue and consent obtained when possible if a baby is deemed eligible.

The initial echocardiogram and Doppler assessment will incorporate:

- Size of the PDA and flow pattern according to standard trial methodology 
- Size of the PDA will be determined at the site of maximum constriction (minimum diameter) using gain optimisation typically at the pulmonary end by determining the average of 3 separate clips

- If the size of the PDA is at least $1.5 \mathrm{~mm}$, flow pattern will be determined by placing the pulse gate in the PDA while adjusting the velocity scale to its highest setting. If the shunt direction is $>1 / 3$ duration of a cycle being right to left, then a rescan will be attempted after a few hours.

If the echocardiogram findings raise concerns about possibility or diagnosis of congenital heart disease, a referral will be made to a paediatric cardiologist as per clinician discretion.

\section{Echocardiograms}

Echocardiograms are performed as part of the normal care of preterm babies. However, clinicians are required to perform an echocardiogram within $72 \mathrm{~h}$ of birth, at around 3 weeks of age (range of 18-24 days) or at discharge from the neonatal unit if discharged before this time.

A proportion of echocardiogram scans will be reviewed by a qualified clinician, who is not involved in recruiting for the trial, to assess consistency between clinicians.

\section{Randomisation and blinding}

Treatment allocation of ibuprofen or placebo are in a ratio of 1:1 and blinded such that the allocation will not be known to clinicians, the baby's family or the trial outcome assessors.

The randomisation system is hosted by the NPEU Clinical Trials Unit. It is accessed by sites via a secure password protected system. If there are problems with accessing the system outside of normal working hours a $24 \mathrm{~h}$ emergency call service is available. The randomisation program uses a minimisation algorithm to ensure balance between the groups. These are allocated with respect to the size of the PDA, gestational age at birth, age at randomisation, sex, trial site, multiple births, mode of respiratory support at randomisation (1) invasive ventilation (by an endotracheal tube); or (2) non-invasive respiratory support through nasal CPAP, nasal ventilation, humidified high flow nasal cannula therapy, or low flow oxygen $\geq 1.1 \mathrm{~L} / \mathrm{min}$; or (3) receiving no mechanical ventilation, or pressure support (in room air, or low flow oxygen $<1.1 \mathrm{~L} / \mathrm{min}$, or ambient oxygen) and whether baby is receiving inotropes at the time of randomisation. Babies of multiple births are randomised individually.
If necessary, the treatment allocation code may be broken for a participant at the request of the site PI or clinician in charge of the baby.

\section{Description of intervention}

Ibuprofen is supplied as a clear sterile solution at a concentration of $5 \mathrm{mg} / \mathrm{ml}$ in ampoules. Cartons contain four $2 \mathrm{ml}$ single use ampoules. Each carton is labelled with a unique code and in compliance with the guidance given in Annexe 13 of the European Commission's guidelines for Good Manufacturing Practice.

An initial loading dose of $10 \mathrm{mg} / \mathrm{kg}(2 \mathrm{ml} / \mathrm{kg})$ of ibuprofen is administered, followed by two $5 \mathrm{mg} / \mathrm{kg}(1$ $\mathrm{ml} / \mathrm{kg}$ ) doses at 24 and $48 \mathrm{~h}$ after the initial dose. Doses are calculated on the birth weight of the baby and preferably administered undiluted. If required, the IMP can be diluted to appropriate volume with $5 \%$ glucose or 0.9\% Sodium Chloride. Each dose should be given as a short intravenous infusion over $15 \mathrm{~min}$. All 3 doses should be given unless there are adverse effects necessitating stoppage. Placebo is supplied as a clear sterile solution of $0.9 \%$ Sodium Chloride for injection. Cartons are identical to those for ibuprofen. Volume of placebo withdrawn from the ampoule is calculated following the calculations for ibuprofen dosing.

Following randomisation, first dose should be administered soon after randomisation, after $6 \mathrm{~h}$ of age and within $72 \mathrm{~h}$ of birth. Detailed accountability records are maintained to document which pack of medication is allocated to which baby.

\section{Discontinuation of intervention and withdrawal}

At all stages of the trial it is made clear to the parent(s) that they remain free to withdraw their baby without the need to provide reason or explanation. If parent(s) choose to withdraw their baby from trial participation, permission will be sought to complete data collection and use data up to the point of withdrawal.

A baby may also be withdrawn from the trial, if deemed by the Principal Investigator to be in their best interests.

\section{Safety reporting}

An independent DMC has been established to review the study data and outcomes including safety reports of Serious Adverse Events (SAEs). The DMC will ensure the safety and wellbeing of the trial participants and make recommendations to the Trial Steering Committee (TSC) regarding continuance of the study or modification of the protocol. The TSC has ultimate responsibility for deciding whether the trial should be stopped on safety grounds.

Relationship of each adverse event to the trial medication is determined by a medically qualified 
individual. Causality of adverse events reported in the preterm newborn is difficult to assess since they may be related to the haemodynamic consequences of the patent ductus arteriosus as well as to direct effects of ibuprofen. In addition to this, high incidences of adverse events are foreseeable due to the nature of the patient population and the routine care/treatment. Consequently, only those adverse events identified as serious and not foreseeable will be recorded under trial safety reporting procedures. Safety reporting for each participant is monitored from first dose until 7 days after trial medication. Unforeseeable SAEs are reported to the NPEU CTU within $24 \mathrm{~h}$ of staff at site becoming aware of the event. SUSARs are reported to the MHRA and the approving Research Ethics Committee (REC) within 7 days, if the event results in death or is life-threatening, and within 15 days for all other SUSARs. In addition, a copy of the SAE form corresponding to the event is forwarded to the Chair of the DMC.

All foreseeable SAEs in the trial population that do not require reporting are specified in the full Protocol available to all sites (Additional file 1).

\section{Definition of end of study}

The end of the trial is defined as the date when the trial database is locked. An end of trial declaration will be made to the MHRA and REC. Guidelines for early cessation of the trial have been agreed with the DMC and documented in the DMC Charter.

\section{Data collection}

The outcome data for this trial are routinely recorded clinical items obtained from the clinical notes. No additional blood or tissue samples are required for this trial. All data is collected using trial specific CRFs. Outcome data is collected until discharge home and at 2 years corrected age.

\section{Project Management}

The trial is sponsored by the University of Oxford and is run by the NPEU CTU, based at the University of Oxford and the Chief Investigator. On a day-to day basis, the trial is supervised by a Project Management Group (PMG) according to NPEU CTU SOPs and is subject to audit and inspection. The core PMG meets every month, either remotely or face-to-face. An extended PMG (Co-Investigator Group) meets regularly to troubleshoot, review progress and forward plan. The PMG reports to the TSC.

The trial is overseen by the TSC which has ultimate responsibility for considering and, as appropriate, acting on the recommendations of the DMC. The TSC includes an independent chair, at least one clinician, statistician and Patient and Public Involvement (PPI) representative, and the Chief Investigator. The TSC meets annually to review the progress of the trial.

The DMC is independent of the study and the TSC. The DMC reviews the progress of the trial and interim analysis at least annually and makes recommendations on the conduct of the trial to the TSC.

\section{Patient and public involvement}

Patient and public representatives have been extensively involved in trial planning, grant/protocol writing, and preparing study materials. Advice from two PPI coapplicants included aspects of protocol design, wording in parent-facing documentation, as well as ongoing recruitment initiatives. Contributions on documentation were also received from Bliss baby charity [9]. A lay person is a member of the TSC and is involved in trial oversight and dissemination of findings.

\section{Statistics and analysis}

Sample size and power calculation

Evidence from the TIPP trial suggests that the risk of death or BPD in extremely low birth weight babies at 36 weeks postmenstrual age allocated placebo is 52\% (95\% CI 48 to $56 \%$ ) [10, 11]. However, this trial investigated the effect of prophylactic treatment and included all babies weighing $500-999 \mathrm{~g}$. More recent information using data derived from the latest report of Neonatal Survey Database from the Trent region [12] provides an approximate rate of death by or BPD at 36 weeks postmenstrual age of $53 \%$ for all babies admitted to the neonatal unit. These babies would have been treated according to clinical judgement and therefore a proportion of them would have been treated with ibuprofen. Given that the risk of death or BPD in babies with an echocardiographically confirmed large PDA is inherently higher, it is estimated that the risk in this group is $60 \%$.

$\mathrm{Su}$ et al. (2008) [3] compared ibuprofen to indomethacin in babies $\leq 28$ weeks' gestation having a PDA who were less than $24 \mathrm{~h}$ old. The combined outcome of death within 30 days or BPD at 36 weeks postmenstrual age was observed to be $42 \%$ (95\% CI 29 to $55 \%)$.

It is therefore expected, given that babies will be enrolled up to $72 \mathrm{~h}$ after birth, that the treatment group incidence of death/BPD at 36 weeks postmenstrual age will be approximately $48 \%$ in the intervention arm. This would imply an absolute risk reduction of $12 \%$ (60 to $48 \%$ ) in the primary outcome of the trial for babies randomised to treatment compared to placebo, which is considered a clinically important difference.

Some babies will require open-label treatment (OLT) in either the treatment or placebo arm. As open-label treatment should be limited to symptomatic babies 
meeting only defined criteria, it is considered to have minimal or no effect on the primary outcome. Thus, adjustment of the sample size for open-label treatment is not considered necessary. A full list of OLT criteria are specified in the full Protocol available to all sites.

Regarding outcomes at 2 years corrected age, assuming the risk of a child dying before two years of age is $10 \%$, questionnaires will be sent out to around 660 parents of surviving children. Assuming an attrition rate of $20 \%$ reduces the sample size to around 530. The proportion of infants surviving to 2 years without moderate or severe neurodevelopmental disability in the control group is expected to be $55 \%$ [13]. With outcome data available on a total sample size of around 600 (including deaths) the trial will have an $80 \%$ power to detect an increase in survival without moderate or severe neurodevelopmental disability of $11 \%$ from 55 to $66 \%$ and $90 \%$ to detect an increase of $13 \%$ from 55 to $68 \%$.

\section{Description of statistical methods}

Babies will be analysed in the groups to which they are randomly assigned, comparing the outcome of all babies allocated to ibuprofen with all those allocated to placebo, regardless of deviation from the protocol or treatment received (referred to as the Intention to Treat (ITT) population).

Baseline characteristics and outcomes will be summarised with counts and percentages for categorical variables, means and standard deviations for normally distributed continuous variables, or median and interquartile range for other non-normally distributed continuous or time-to-event variables.

For binary outcomes, risk ratios and confidence intervals will be calculated using log binomial regression, or if a model fails to converge a Poisson regression model with a robust variance estimator will be used. Continuous outcomes will be analysed using linear regression models, with mean differences and confidence intervals presented for approximately normally distributed outcomes. Skewed continuous outcomes will be analysed using quantile regression models, with median differences and confidence intervals presented. Time-to-event outcomes will be analysed using Cox regression and hazard ratios with confidence intervals will be presented.

Analyses will be adjusted for all minimisation factors and the correlation between siblings from multiple births where possible. Both crude and adjusted effect estimates will be presented, but the primary inference will be based on the adjusted estimates.

Due to the multiple number of short-term outcomes, and correlation between some outcomes, statistical inference will be restricted to a predefined list of tested outcomes. Summary data by trial arm will be provided for all other outcomes, but statistical tests (or the calculation of confidence intervals) will not be performed.

Full details of the statistical analysis will be documented in the Statistical Analysis Plan.

\section{Discussion}

Permitted and non-permitted medications

All prescribed medications deemed necessary to provide adequate supportive care to the baby, are permitted at any stage during the trial period. However, open treatment with indomethacin or ibuprofen or other nonsteroidal anti-inflammatory drugs (NSAIDs) should be avoided unless the criteria for open-label treatment are met.

The concomitant administration of other medication is not restricted but should be closely monitored for an interaction by the treating clinician.

\section{Open-label treatment}

If the clinical condition of a baby warrants intervention, open-label treatment can be given to close the PDA (medical or surgical). The following criteria, however, have been devised to limit and rationalise the use of open-label treatment but it is recognised that clinicians may need to override this guidance in the best interests of the baby. Clinical responsibility for the care of the baby will remain fully with the neonatal clinical team irrespective of the trial.

To consider open label (Rescue) treatment, both clinical AND echocardiographic criteria as described below should be met:

1. Clinical findings of inability to wean on ventilator (ventilated for at least 7 days continuously) AND inability to wean oxygen

OR

Persistent hypotension and/or pulmonary haemorrhage and/or signs of cardiac failure

2. Echocardiographic findings of a large PDA (PDA $\geq$ $2.0 \mathrm{~mm}$ with pulsatile flow)

\section{AND}

Hyperdynamic circulation and/or ductal steal (please refer to Baby-OSCAR ECHO workbook).

\section{Participant confidentiality and retention of personal data}

Contact details of the baby's parent(s), as well as the baby's name and any other identifying details is collected via CRFs. Parents of babies participating in the trial are informed of and provide consent to this.

Overall responsibility for ensuring that each participant's information is kept confidential lies with the Sponsor. All paper documents are stored securely and kept in strict confidence in compliance with current data regulations. Data collected on the CRFs are stored in an electronic database held by the Trial Coordinating 
Centre in which the participant is identified only by a trial specific number.

Personal data are needed to contact parents when their children are 2 years of age, to co-ordinate follow up, and to disseminate the results of the trial to parent(s). Personal data is held securely and will not be used for any other purpose.

Following trial completion and report publication, data will be archived in a secure physical and electronic location with restricted access.

\section{Participant remuneration}

A $£ 15$ voucher is sent to parents as an incentive to complete the 2-year follow-up questionnaire; however its completion is not dependent on receiving the voucher.

\section{Dissemination}

The success of the trial depends on a large number of neonatal nurses, neonatologists, and parent(s). Credit for the trial findings will be given to all who have collaborated and participated in the trial including all local co-ordinators and collaborators, members of the trial committees, the Baby-OSCAR Co-ordinating Centre and trial staff. Authorship at the head of the primary results paper will take the form "[name], [name] and [name] on behalf of the 'The Baby-OSCAR Collaborative Group"'. All contributors to the trial will be listed at the end of the main paper, with their contribution identified.

It is the intention of the Baby-OSCAR Collaborative Group to present data at national/international conferences, and three open-access peer-reviewed articles including the analysis of key outcomes. The NPEU Clinical Trials Unit will disseminate the results at conferences and coordinate press releases, website promotion, social and other media interest. Parents will be sent a summary of trial publications if they wish.

\section{Supplementary Information}

The online version contains supplementary material available at https://doi. org/10.1186/s12887-021-02558-7.

\section{Additional file 1. \\ Additional file 2. \\ Additional file 3.}

\section{Abbreviations}

AE: Adverse Event; AR: Adverse Reaction; BPD: Bronchopulmonary Dysplasia; $\mathrm{Cl}$ : Confidence Interval; COX: Cyclo-oxygenase; CPAP: Continuous Positive Airway Pressure; DA: Ductus Arteriosus; DMC: Data Monitoring Committee; ECHO: Echocardiography; GP: General Practitioner; HRA: Health Research Authority; HTA: Health Technology Assessment; ICF: Informed Consent Form; ICH: International Conference on Harmonisation; IMP: Investigational Medicinal Product; IRAS: Integrated Research Application System; ITT: Intention to Treat; IVH: Intraventricular Haemorrhage; MHRA: Medicines and Healthcare products Regulatory Agency; NEC: Necrotising Enterocolitis; NHS: National Health Service; NIHR: National Institute for Health Research;
NPEU CTU: National Perinatal Epidemiology Unit Clinical Trials Unit; NSAI D: Non-Steroidal Anti-inflammatory Drug; OR: Odds Ratio; PARCA-R: Parent Report of Cognitive Abilities-Revised; PDA: Patent Ductus Arteriosus; PIL: Parent Information Leaflet; PMA: Postmenstrual Age; PMG: Project Management Group; PVL: Cystic Periventricular Leukomalacia; REC: Research Ethics Committee; RDS: Respiratory Distress Syndrome; ROP: Retinopathy of Prematurity; SAE: Serious Adverse Event; SAR: Serious Adverse Reaction; SUSAR: Suspected Unexpected Serious Adverse Reaction; TSC: Trial Steering Committee

\section{Acknowledgements}

We are grateful to the parents of participating infants and staff and carers in recruiting and continuing care sites. We thank the members of the independent DMC and the TSC and the administrative and support colleagues at the NPEU Clinical Trials Unit, including Andrew King, David Murray, Ursula Bowler, Christina Cole, Charles Roehr, Nina Jamieson and Julia Sutton. The authors would also like to thank the Bliss charity for their contributions towards parent-facing documents and the Research and Development department of North Tees hospital for their help and support.

\section{Recruitment status}

Ongoing: at the time of submission for publication, participants are currently being recruited and enrolled to Baby-OSCAR. Information regarding study sites can be found on the Baby-OSCAR website [14].

\section{Funding and sponsorship}

This study is funded by the National Institute for Health Research (NIHR) Health Technology Assessment programme (11/92/15). The views expressed are those of the author(s) and not necessarily those of the NIHR or the Department of Health and Social Care. The funder provided advice and support and monitored study progress but did not have a role in study design or data collection, analysis and interpretation.

Baby-OSCAR is sponsored by the University of Oxford. The contact on behalf of the sponsor for all correspondence relating to applications for this project is Clinical Trials \& Research Governance, ctrg@admin.ox.ac.uk.

\section{Trial steering committee}

The independent members of the TSC are Emeritus Professor Michael Weindling (Chair), Professor Denis Azzopardi, Professor Narendra Aladangady (from 2018), Professor Tim Clayton, Mrs. Sophie Welch (PPI), Mr. Ben Snook (PPI) and Dr. Eleri Adams (stepped down in 2018).

The non-independent members are Professor Samir Gupta and Professor Edmund Juszczak.

\section{Data monitoring committee}

The independent members of the DMC are Professor David Edwards (Chair), Professor Alan Montgomery and Professor Heike Rabe.

\section{Declaration of Helsinki}

The Chief Investigator will ensure that this study is conducted in accordance with the principles of the Declaration of Helsinki.

\section{Guidelines for good clinical practice}

The Chief Investigator will ensure that this study is conducted in accordance with relevant regulations and with Good Clinical Practice.

\section{Authors' contributions \\ SG conceived the idea for the study and wrote the initial protocol, with clinical/scientific contributions from EJ, PH, SJ, TR, NS, JW, DF, SS, WK and PPI input from EH and JP. SG is the Chief Investigator responsible for all aspects of the study, including preparation and submission of the grant application, securing funding, regulatory approvals, project management, data collection and preparation of the manuscript for publication. EJ and $\mathrm{PH}$ provided methodological expertise to design and refine the study protocol. JB and $\mathrm{LL}$ provided statistical and methodological expertise, and KS, ML, and CE contributed logistical aspects of the trial. All authors approved the final version of the manuscript.}

\section{Availability of data and materials}

All data requests must be submitted to the corresponding author for consideration. Please note exclusive of data use will be retained until the 
publication of major outputs. Access to anonymised data may be granted following review of request.

\section{Ethics approval and consent to participate}

Baby-OSCAR received approval from the MHRA, the NHS Health Research Authority, and East Midlands - Nottingham 2 Research Ethics Committee: nottingham2.rec@hra.nhs.uk on 16 December 2014 (REC ref.: 14/EM/0172). Trust Confirmation of Capacity and Capability was obtained at each site. The Chief Investigator or delegate will submit an Annual Progress Report, End of Study Notification, and Final Report to the Funder; NIHR HTA, MHRA, REC, HRA, host organisation and Sponsor.

Where necessary, approvals are obtained from the above parties for all substantial amendments to the original approved documents and disseminated by NPEU CTU to relevant parties.

Parents of eligible babies are provided with both verbal and written information in the form of a Parent Information Leaflet (Additional file 2), by their clinical team. Parent(s) with legal parental responsibility of eligible babies are approached to discuss the trial further, answer any questions they may have, and to request consent. Parent(s) have as much time as they need to consider the information, and the opportunity to question the research team, or other independent parties to decide whether they wish to participate in the study. Informed consent for the study must obtained by a suitably qualified member of the study team. A parent must personally sign and date the latest approved version of the Informed Consent form (Additional file 3) before any study specific procedures are performed. Parent(s) who do not speak English will only be approached if an adult interpreter is available.

\section{Consent for publication}

Not applicable.

\section{Competing interests}

SG is member of steering committee of ESPR NPE (Neonatologist performed echocardiography). EJ was a member of the NIHR HTA General Board from 2016 to 2017 and the NIHR HTA Commissioning Board from 2013 to 2016. $\mathrm{PH}$ is currently a member of the NIHR HTA Commissioning Board. EJ, LL, NS and $\mathrm{PH}$ report receipt of funding from NIHR, outside the submitted work. NS receives fees for educational activities from Recordati Group.

\section{Author details}

${ }^{1}$ University Hospital of North Tees, Hardwick Road, Stockton-On-Tees TS19 8PE, UK. ${ }^{2}$ National Perinatal Epidemiology Unit (NPEU) Clinical Trials Unit, Nuffield Department of Population Health, University of Oxford, Old Road Campus, Headington, Oxford OX3 7LF, UK. ${ }^{3}$ Nottingham Clinical Trials Unit, School of Medicine, University of Nottingham, University Park Nottingham, Nottingham NG7 2RD, UK. ${ }^{4}$ Institute of Applied Health Research, University of Birmingham, Birmingham B15 2TT, UK. 'Liverpool Women's NHS Foundation Trust, Crown Street, Liverpool L8 7SS, UK. ${ }^{6}$ South Tees Hospitals NHS Foundation Trust, James Cook University Hospital, Middlesbrough TS4 3BW, UK. ${ }^{7} \mathrm{NICU}$, Rosie Hospital, Cambridge University Hospital Foundation Trust, Cambridge CB2 2QQ, UK. ${ }^{8}$ The University of Leicester, Department of Health Science, University Road, George Davies Centre, Leicester LE1 7RH, UK.

Received: 18 December 2020 Accepted: 15 February 2021

Published online: 26 February 2021

\section{References}

1. Nemerofsky SL, Parravicini E, Bateman D, Kleinman C, Polin RA, Lorenz JM. The ductus arteriosus rarely requires treatment in infants $>1000$ grams. Am J Perinatol. 2008;25(10):661-6. https://doi.org/10.1055/s-0028-1090594 Epub 2008 Oct 10

2. Noori S, McCoy M, Friedlich P, Bright B, Gottipati V, Seri I, Sekar K. Failure of ductus arteriosus closure is associated with increased mortality in preterm infants. Pediatrics. 2009;123:138-e144.

3. Noori S. Patent ductus arteriosus in the preterm infant: to treat or not to treat? J Perinatol. 2010;30(Suppl):S31-7.

4. Su BH, Lin HC, Chiu HY, Hsieh HY, Chen HH, Tsai YC. Comparison of ibuprofen and indomethacin for early-targeted treatment of patent ductus arteriosus in extremely premature infants: a randomised controlled trial. Arch Dis Child Fetal Neonatal Ed. 2008;93(2):F94-9.
5. Oncel MY, Yurttutan S, Degirmencioglu H, Uras N, Altug N, Erdeve O, Dilmen $U$. Intravenous paracetamol treatment in the management of patent ductus arteriosus in extremely low birth weight infants. Neonatology. 2013:103:166-9.

6. Sellmer A, Vandborg Bjerre J, Schmidt MR, McNamara PJ, Hjortdal VE, Host $\mathrm{B}$, Bech $\mathrm{BH}$, Henriksen TB: Morbidity and mortality in preterm neonates with patent ductus arteriosus on day 3. Arch Dis Child Fetal Neonatal Published online first 26 July 2013; https://doi.org/10.1136/archdischild-2013-303816.

7. Clyman RI. The role of patent ductus arteriosus and its treatment in the development of bronchopulmonary dysplasia. Semin Perinatol. 2013;37:1027.

8. Johnson S, Bountziouka V, Linsell L, Brocklehurst P, Marlow N, Wolke D, Manktelow B. Parent report of Children's abilities-revised (PARCA-R). Technical and Interpretive Manual. Leicester: University of Leicester; 2019.

9. Bliss - for babies born premature or sick: www.bliss.org.uk. Accessed $8 \mathrm{Dec}$ 2020.

10. Schmidt B, Davis P, Moddemann D, Ohlsson A, Roberts RS, Saigal S, et al. Long-term effects of indomethacin prophylaxis in extremely-low-birthweight infants. N Engl J Med. 2001;344(26):1966-72.

11. Schmidt B, Roberts RS, Fanaroff A, Davis P, Kirpalani HM, Nwaesei C, et al. Indomethacin prophylaxis, patent ductus arteriosus, and the risk of bronchopulmonary dysplasia: further analyses from the trial of indomethacin prophylaxis in Preterms (TIPP). J Pediatr. 2006;148(6):730-4.

12. Neonatal Survey Database from the Trent Region 2010; https://www.le.ac uk/departments/health-sciences/research/timms/projects/tns. Accessed 8 Dec 2020.

13. Mangham LJ, Petrou S, Doyle LW, Draper ES, Marlow N. The cost of preterm birth throughout childhood in England and Wales. Pediatrics. 2009;123(2): e312-27.

14. Baby-OSCAR study: https://www.npeu.ox.ac.uk/baby-oscar. Accessed 3 Dec 2020.

\section{Publisher's Note}

Springer Nature remains neutral with regard to jurisdictional claims in published maps and institutional affiliations.

\section{Ready to submit your research? Choose BMC and benefit from:}

- fast, convenient online submission

- thorough peer review by experienced researchers in your field

- rapid publication on acceptance

- support for research data, including large and complex data types

- gold Open Access which fosters wider collaboration and increased citations

- maximum visibility for your research: over $100 \mathrm{M}$ website views per year

At $\mathrm{BMC}$, research is always in progress.

Learn more biomedcentral.com/submission 